\title{
Dimetilfumarato. Una alternativa terapéutica en la psoriasis
}

\section{Dimethyl fumarate. A therapeutic alternative in psoriasis}

\author{
David Aldo De Luca
}

\section{RESUMEN}

Los ésteres de ácido fumárico se utilizan de forma empirica desde hace décadas para el manejo de la psoriasis moderada a grave que requiere un tratamiento sistémico. Tanto su efecto inmunomodulador como su baja tasa de efectos adversos graves lo hacen atractivo como opción terapéutica en los pacientes que no responden a otros tratamientos o que tienen algunas contraindicaciones para recibir las terapéuticas habituales. Se presenta una actualización bibliográfica del dimetilfumarato en el tratamiento de la psoriasis moderada a grave y se destacan su mecanismo de acción, posología, efectos indeseados y los estudios que avalan su utilización en la psoriasis. Palabras clave: dimetilfumarato, ésteres de ácido fumárico, psoriasis.

Dermatol. Argent. 2020, 26 (3): 114-120

\section{ABSTRACT}

Fumaric acid esters have been empirically used for decades for the management of moderate to severe psoriasis that requires systemic treatment. Both its immunomodulatory effect and its low rate of serious adverse effects make it attractive as a therapeutic option in patients who do not respond to other treatments or who have certain contraindications to the usual therapies. A bibliographic update on dimethyl fumarate in the treatment of moderate to severe psoriasis is presented, highlighting its mechanism of action, its dosage, its unwanted effects and the studies that support its use in psoriasis.

Key words: dimethyl fumarate, fumaric acid esters, psoriasis.

Dermatol. Argent. 2020, 26 (3): 114-120
Médico Especialista en Dermatología, M.Sc. Biología Molecular Consultorio Privado, Bad Neustadt a.d. Saale, Baviera, Alemania
Contacto autor: David A. De Luca E-mail: daviddeluca@gmail.com Fecha de trabajo recibido: $3 / 6 / 2020$ Fecha de trabajo aceptado: 24/8/2020 Conflicto de interés: el autor declara que no existe conflicto de interés.

\section{INTRODUCCIÓN}

La psoriasis es una enfermedad inflamatoria sistémica crónica, de etiología multifactorial, que afecta a sujetos genéticamente predispuestos. La afectación de la piel y las faneras es característica y en la actualidad hay una gran variedad de opciones terapéuticas. A continuación, se presenta una revisión bibliográfica sobre el dimetilfumarato (DMF), una medicación antipsoriática escasamente difundida en Argentina. Se consultaron los Consensos Europeos de Tratamiento en la Psoriasis y las bases de datos internacionales Medline mediante PubMed y Scholar Google, donde se emplearon las palabras "dimetilfumarato", "ésteres de ácido fumárico", "Skilarence" y "Fumaderm", se limitaron los resultados a la enfermedad psoriasis y se seleccionaron solo los artículos en español, inglés o alemán.

\section{¿QUÉ ES EL ÁCIDO FUMÁRICO?}

El ácido fumárico es un ácido dicarboxílico insaturado de color cristalino cuya fórmula química es $\mathrm{C}_{4} \mathrm{H}_{4} \mathrm{O}_{4}$ y es un compuesto endógeno intermediario del ciclo del ácido tricarboxílico (ciclo de Krebs). En la naturaleza, este ácido orgánico se encuentra en la hierba Fumaria officinalis, vulgarmente conocida como palomilla, fumaria o sangre de Cristo ${ }^{1}$. Este herbáceo se distribuyó originariamente en Europa y se importó más tarde a América del Norte. En 1959, el químico alemán Walter Schweckendiek lo utilizó en el tratamiento de su propia psoriasis cutánea. Él interpretó, erróneamente, que en dicha enfermedad había una deficiencia de ácido fumárico dentro del ciclo de Krebs y que su reposición produciría una mejoría de las manifestaciones cutáneas ${ }^{2}$. 
Dada la buena respuesta cutánea que Schweckendiek experimentó, el ácido fumárico se utilizó de manera empírica entre las décadas de 1970 y 1990 en Alemania, Suiza y los Países Bajos. En 1994, fue aprobado como tratamiento de la psoriasis vulgar grave en Alemania y recomendado en las guías europeas para el tratamiento sistémico de esa enfermedad ${ }^{2,3}$. El DMF también se utilizó con fines no medicinales por su acción antibacteriana contra E. coli, como inhibidor del crecimiento de mohos en el pan y fungicida para muebles, zapatos y ropa, pero debido a su alta tasa de dermatitis de contacto alérgica (dermatitis de sofá y del calzado), se lo retiró del mercado europeo en $2009^{4}$.

\section{INDICACIONES Y COMPOSICIÓN DEL DIMETILFUMARATO}

Los ésteres de ácido fumárico (EAF) están aprobados en Alemania para el tratamiento de la psoriasis desde 1994 (Fumaderm ${ }^{\odot}$ ), pero por sus efectos adversos, a partir de 2008 solo están aprobados para las formas más graves ${ }^{2}$. Fumaderm ${ }^{\oplus}$ contiene diversos EAF como la droga activa DMF, un diéster de ácido fumárico, junto con las sales de monoetilfumarato de calcio, de cinc y de magnesio. La forma pura de DMF sin sales de monoetilfumarato (Skilarence ${ }^{\ominus}$ ) se comercializa en Europa desde 2017 y está indicada para el tratamiento de la psoriasis en placas moderada o grave que requiere un tratamiento sistémico en pacientes mayores de 18 años 5 . La composición de los comprimidos de Fumaderm $^{\oplus}$ y de Skilarence ${ }^{\oplus}$ se detalla en la Tabla $1^{6}$. Respecto del DMF en la artritis psoriásica, no existe indicación formal ni está aprobado para su utilización. Sin embargo, algunos autores describen una mejoría del dolor articular en comparación con el uso del placebo ${ }^{7}$.

Por su efecto inmunomodulador, también está aprobado en Estados Unidos para el tratamiento de las recaídas en la esclerosis múltiple remitente-recurrente desde 2013 (Tecfidera $^{\circledR}$ ). En la Argentina, Tilmurato ${ }^{\circledR}$ en cápsulas de 120 y $240 \mathrm{mg}$ se encuentra aprobado por la

\begin{tabular}{|l|c|c|c|}
\hline \multicolumn{1}{|c|}{ Composición } & $\begin{array}{c}\text { Fumaderm }^{\odot} \\
\mathbf{3 0} \mathrm{mg}\end{array}$ & $\begin{array}{c}\text { Fumaderm }^{\odot} \\
120 \mathrm{mg}\end{array}$ & $\begin{array}{c}\text { Skilarence }^{\odot} \\
120 \mathrm{mg}\end{array}$ \\
\hline Dimetilfumarato & $30 \mathrm{mg}$ & $120 \mathrm{mg}$ & $120 \mathrm{mg}$ \\
\hline $\begin{array}{l}\text { Monoetilfumarato } \\
\text { de calcio }\end{array}$ & $67 \mathrm{mg}$ & $87 \mathrm{mg}$ & - \\
\hline $\begin{array}{l}\text { Monoetilfumarato } \\
\text { de magnesio }\end{array}$ & $5 \mathrm{mg}$ & $5 \mathrm{mg}$ & - \\
\hline $\begin{array}{l}\text { Monoetilfumarato } \\
\text { de cinc }\end{array}$ & $3 \mathrm{mg}$ & $3 \mathrm{mg}$ & - \\
\hline TABLA 1: Composición química de Fumaderm ${ }^{\circledR}$ y Skilarence & \\
\hline
\end{tabular}

ANMAT desde 2016 y se comercializa sólo para el tratamiento de dicha enfermedad neurológica ${ }^{8}$. Los EAF se han utilizado con indicación off-label en el tratamiento de otras enfermedades dermatológicas, como la necrobiosis lipídica, el granuloma anular y la sarcoidosis?.

\section{CARACTERÍSTICAS FARMACOCINÉTICAS DEL DIMETILFUMARATO}

El DMF se administra por vía oral, pero su tasa de absorción gastrointestinal es muy baja. Las estearasas presentes en el medio alcalino intestinal hidrolizan el DMF en monometilfumarato (MMF), el metabolito activo. El pico plasmático de MMF se observa entre las 2 y 2,5 horas luego de su ingesta y la administración de DMF junto con los alimentos ricos en ácidos grasos lo retrasa. Rastros de DMF pueden encontrarse en la sangre, pero no presentan afinidad a las proteínas plasmáticas, a diferencia de lo que ocurre con MMF, que alcanza una unión cercana al 50\%. Aun con dosis altas de la medicación, es decir $240 \mathrm{mg}$ tres veces por día, no se observa acumulación de MMF y las concentraciones del fármaco descienden rápidamente luego de su suspensión. Por ese motivo, se recomienda un intervalo no mayor de 12 horas entre cada administración de $\mathrm{DMF}^{5,10}$.

La metabolización de DMF, como también la de MMF, es independiente de las enzimas del citocromo $\mathrm{P}-450$, por lo que no se describieron interacciones medicamentosas. El DMF reacciona con el glutatión para formar el ácido mercaptúrico que se excreta por la orina. El MMF se metaboliza a ácido fumárico, ingresa en el ciclo del ácido tricarboxílico y finalmente se degrada en agua y dióxido de carbono, excretados por vía respiratoria y, en menor medida, por vía renal y digestiva. Por todas las características farmacocinéticas hasta aquí nombradas, el DMF es seguro en los pacientes con insuficiencia renal y hepática leve a moderada y no requiere ajuste de la dosis en dichas situaciones ${ }^{5,11}$.

\section{MECANISMOS DE ACCIÓN}

Si bien los mecanismos de acción de DMF no se encuentran del todo dilucidados, se destacan como fundamentales los efectos antiinflamatorios, antioxidantes e inmunomoduladores. Algunas acciones dependen meramente de DMF y otras, de $\mathrm{MMF}^{6}$. A continuación, se describen los mecanismos moleculares (Gráfico 1) y celulares (Tabla 2) propuestos para su acción en la psoriasis ${ }^{9}$ :

a) Interacción de DMF con el glutatión intracelular: la depleción de glutatión mediante su unión a DMF y MMF induce indirectamente la activación del factor de transcripción Nrf2 y la síntesis de hemooxigenasa 1 , con efecto antiinflamatorio y citoprotector contra el estrés oxidativo ${ }^{13}$. 


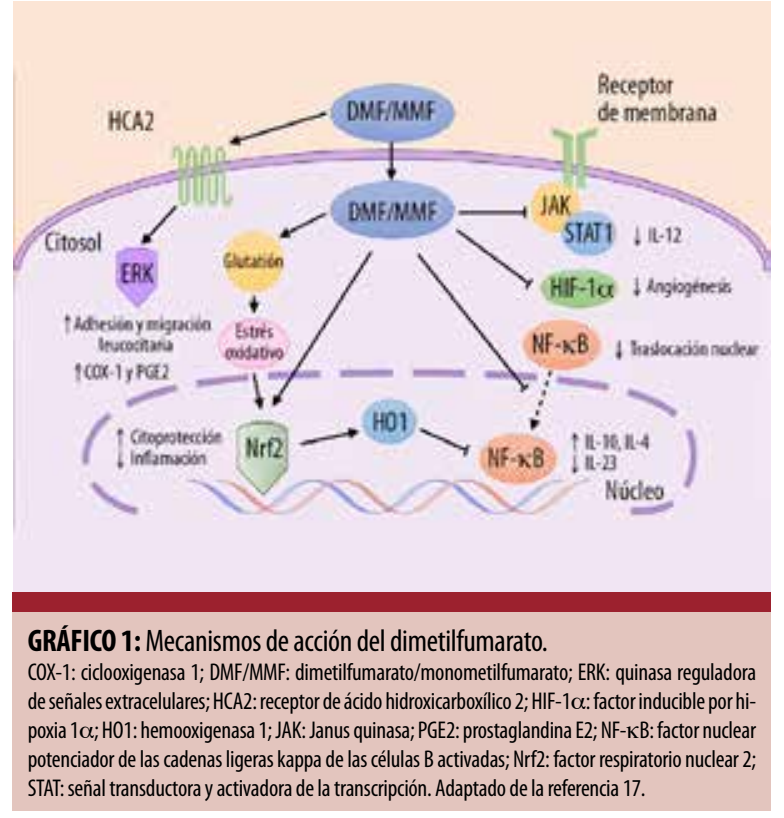

\begin{tabular}{|c|c|}
\hline Célula & Efecto \\
\hline Neutrófilo & $\begin{array}{l}\text { Reducción de la activación celular, de la circulación y } \\
\text { de la migración transendotelial }\end{array}$ \\
\hline Linfocito $T$ & $\begin{array}{l}\text { Inducción de la apoptosis y linfopenia } \\
\text { Reducción de las células T de memoria } \\
\text { Aumento de las células T reguladoras } \\
\text { Aumento de las células Th2, reducción del perfil Th1 y Th17 }\end{array}$ \\
\hline Linfocito B & $\begin{array}{l}\text { Inducción de la apoptosis } \\
\text { Reducción de los linfocitos B CD19 }\end{array}$ \\
\hline $\begin{array}{l}\text { Célula } \\
\text { dendrítica }\end{array}$ & $\begin{array}{l}\text { Inducción de la apoptosis, expresión de genes cito- } \\
\text { protectores y reducción de la maduración celular } \\
\text { Formación de células dendríticas de tipo II y reduc- } \\
\text { ción de las de tipo I y plasmocitoide } \\
\text { Aumento de la síntesis de IL-10 } \\
\text { Represión génica de IL-12 e IL-23 }\end{array}$ \\
\hline $\begin{array}{l}\text { Célula } \\
\text { endotelial }\end{array}$ & $\begin{array}{l}\text { Reducción de la proliferación celular mediada por } \\
\text { VEGF } \\
\text { Represión de la síntesis de moléculas de adhesión } \\
\text { Bloqueo de la angiogénesis }\end{array}$ \\
\hline $\begin{array}{l}\text { Querati- } \\
\text { nocito }\end{array}$ & $\begin{array}{l}\text { Reducción de la proliferación celular } \\
\text { Aumento del calcio intracelular } \\
\text { Reducción de la expresión de moléculas de adhesión } \\
\text { y de HLA-DR }\end{array}$ \\
\hline Microglía & $\begin{array}{l}\text { Respuesta antioxidante y modulación de la respues- } \\
\text { ta neuroprotectora }\end{array}$ \\
\hline Neuronas & $\begin{array}{l}\text { Reducción de la apoptosis } \\
\text { Supervivencia de las neuronas bajo estrés oxidativo }\end{array}$ \\
\hline $\begin{array}{l}\text { Células } \\
\text { tumorales }\end{array}$ & $\begin{array}{l}\text { Reducción de la proliferación tumoral en el melanoma } \\
\text { Inducción de la apoptosis tumoral }\end{array}$ \\
\hline
\end{tabular}

b) Inhibición de la actividad de NF-кB: DMF inhibe la translocación nuclear de NF- $\kappa \mathrm{B}$, con el consiguiente bloqueo de la cascada inflamatoria y la síntesis de quimioquinas y de moléculas de adhesión ${ }^{14}$. Así, y junto con las acciones de Nrf, DMF induce el viraje linfocitario Th1 y Th17 al perfil Th2, mediante la supresión de la síntesis de las citoquinas IL-1 $\beta$, IL-22, IL-36 $\alpha$ e IL-36 $\gamma$, y la inducción de IL-10 e IL-37 ${ }^{15}$. A nivel histológico, la inhibición de NF- $\mathrm{KB}$ mediante DMF reduce el grosor epidérmico debido a su efecto antiproliferativo ${ }^{16}$.

c) Activación del receptor HCA2: este receptor acoplado a una proteína $G$ se encuentra sobreexpresado en la psoriasis y presenta un efecto regulador de la inflamación. El MMF es un agonista de HCA2 y, mediante la activación de ERK, reduce la adhesión, la migración y el reclutamiento de neutrófilos en la piel. Simultáneamente, induce la producción de ciclooxigenasa 1 y de prostaglandina E2 mediada por niacina en los queratinocitos, lo cual desencadena rubefacción facial, uno de los efectos adversos más frecuentes ${ }^{17}$.

d) Degradación de HIF-1 $\alpha$ : HIF-1 $\alpha$ se encuentra sobreexpresado en la psoriasis y dicho factor de transcripción promueve la síntesis de IL-8 y VEGF. Al promover la degradación de HIF-1 $\alpha$, DMF muestra una acción antiangiogénica ${ }^{18}$.

e) Acciones sobre señales dependientes de JAK/STAT: las IL-12 y 23 son esenciales en la fisiopatogenia de la psoriasis. El DMF promueve la apoptosis de células dendríticas productoras de IL-12, mediante la inactivación de JAK/STAT ${ }^{12}$. Por otro lado, la activación de JAK/STAT3 en dichas células regula de forma negativa la transcripción de IL-23. Este efecto se suma al poder condroprotector de DMF mediante la inhibición de ciertas metaloproteinasas vía JAK/STAT, lo que conlleva a una reducción de la degradación del colágeno $\mathrm{II}^{19}$.

\section{DOSIFICACIÓN RECOMENDADA EN LA PSORIASIS}

En el mercado europeo, Skilarence y Fumaderm $^{\oplus}$ se comercializan en comprimidos orales de $30 \mathrm{mg}$ y de 120 mg de DMF. La dosificación debe ser personalizada y la posología se adapta a las necesidades de cada paciente. La dosis recomendada inicial es de $30 \mathrm{mg} /$ día por la noche durante una semana. La medicación debe ingerirse con un vaso de agua, durante o después de las comidas y se recomienda acompañarla de productos lácteos. Luego, se aumenta la dosis según el esquema indicado en la Tabla $3^{5}$.

Si se obtiene una mejoría clínica antes de alcanzar los $720 \mathrm{mg}$ diarios, puede mantenerse esa posología y no se requiere un aumento de la dosificación. La mejoría se observa típicamente entre la cuarta y la sexta 
semana de tratamiento, aunque se sugiere esperar por lo menos 3 meses antes de considerar que la medicación no ha sido eficaz. Si se logra una mejoría clínica sostenida con $720 \mathrm{mg}$, puede reducirse gradualmente hasta alcanzar una dosis de mantenimiento bien tolerada para el paciente. En caso de efectos adversos, puede reducirse la medicación a la última dosis mejor tolerada y aumentarla de forma gradual ${ }^{5}$.

En la actualidad, se prefiere Skilarence ${ }^{\circledast}$ en lugar de Fumaderm $^{\oplus}$, dado que el primero requiere sólo controles de laboratorio trimestrales. En los pacientes bajo tratamiento crónico con Fumaderm ${ }^{\circledast}$, puede rotarse a Skilarence ${ }^{\circledast}$ dosis equivalentes, sin alteración en la eficacia.

\begin{tabular}{|c|c|c|c|c|}
\hline Semana & Desayuno & Almuerzo & Cena & $\begin{array}{r}\text { Dosis } \\
\text { total }\end{array}$ \\
\hline 1 & ---- & ---- & $30 \mathrm{mg}$ & $30 \mathrm{mg}$ \\
\hline 2 & $30 \mathrm{mg}$ & ---- & $30 \mathrm{mg}$ & $60 \mathrm{mg}$ \\
\hline 3 & $30 \mathrm{mg}$ & $30 \mathrm{mg}$ & $30 \mathrm{mg}$ & $90 \mathrm{mg}$ \\
\hline 4 & ---- & ---- & $120 \mathrm{mg}$ & $120 \mathrm{mg}$ \\
\hline 5 & $120 \mathrm{mg}$ & ---- & $120 \mathrm{mg}$ & $240 \mathrm{mg}$ \\
\hline 6 & $120 \mathrm{mg}$ & $120 \mathrm{mg}$ & $120 \mathrm{mg}$ & $360 \mathrm{mg}$ \\
\hline 7 & $120 \mathrm{mg}$ & $120 \mathrm{mg}$ & $240 \mathrm{mg}$ & $480 \mathrm{mg}$ \\
\hline 8 & $240 \mathrm{mg}$ & $120 \mathrm{mg}$ & $240 \mathrm{mg}$ & $600 \mathrm{mg}$ \\
\hline $9+$ & $240 \mathrm{mg}$ & $240 \mathrm{mg}$ & $240 \mathrm{mg}$ & $720 \mathrm{mg}$ \\
\hline TABLA 3: Dosificación estándar del dimetilfumarato. & \\
\hline & & & &
\end{tabular}

\section{PARÁMETROS DE LABORATORIO PARA CONTROLAR}

Antes de comenzar el tratamiento con Skilarence ${ }^{\bullet}$, debe descartarse el embarazo, el hemograma basal debe presentar $\geq 3.000$ leucocitos $/ \mathrm{ml}$ en la sangre periférica con $\geq 1.000$ linfocitos/ml y se deben controlar las funciones hepática (TGO, TGP, GGT, fosfatasa alcalina y bilirrubina) y renal (urea, creatinina y descartar proteinuria o albuminuria). Si bien no existen valores de corte claros, las alteraciones renales y hepáticas leves a moderadas no contraindican la utilización de DMF y tampoco requieren un ajuste de la dosis. Se recomienda el control trimestral del hemograma y de las funciones renal y hepática. En relación con los resultados, se sugieren las siguientes precauciones:

- Recuento de linfocitos entre 700 y $1.000 / \mathrm{ml}$ : control mensual del hemograma hasta obtener dos valores consecutivos de linfocitos mayores de $1.000 / \mathrm{ml}$.

- Recuento de linfocitos $<700 / \mathrm{ml}$ y/o leucocitos $<3.000 / \mathrm{ml}$ : repetir el hemograma y, si se confirma el recuento, suspender la medicación.
- Eosinofilia: se observa con frecuencia entre la quinta y la octava semana de tratamiento, con posterior normalización. No requiere corrección de la dosis ${ }^{5}$.

- En caso de anemia o trombocitopenia por debajo de $150.000 / \mathrm{ml}$ : suspender transitoriamente la medicación y descartar otras causas.

- Función renal: se indica la suspensión de DMF con aumento de la creatinina mayor del 30\% del valor basal, con control semanal de la función renal. Con aumentos menores del $30 \%$ o proteinuria significativa y sostenida, se indica la reducción de la dosis de DMF y, en caso de persistencia, la suspensión del tratamiento.

- Función hepática: TGP y TGO $<\times 3$ veces el valor de corte, deben descartarse factores asociados hepatotóxicos. La presencia de ictericia o de valores de TGP y TGO $>\times 3$ obliga a la reducción de la dosis de DMF $y$, en caso de persistencia, a suspender el tratamiento ${ }^{20}$.

\section{EFECTOS ADVERSOS ASOCIADOS AL DIMETILFUMARATO}

Los efectos adversos típicos son los trastornos gastrointestinales en más del $60 \%$ de los pacientes y, en menor medida, las alteraciones del hemograma como la linfopenia y la leucopenia leves, de fácil manejo mediante la reducción de la dosis. La frecuencia de presentación de los efectos adversos de DMF se describen en la Tabla $44^{3,5}$.

La rubefacción facial o flushing se presenta en el 20\% de los pacientes, sobre todo al inicio del tratamiento. La rubicundez se asocia a cefalea y puede atenuarse mediante la reducción de la dosis matutina o la administración oral de ácido acetilsalicílico ${ }^{5,10}$.

De manera infrecuente, se destacan el exantema macular y la elevación de las enzimas hepáticas. En particular, los efectos adversos renales como la proteinuria pueden incrementarse con el uso de medicación nefrotóxica concomitante como el metotrexato, la ciclosporina, los agentes citotóxicos, los aminoglucósidos, los diuréticos, los AINE y el litio, entre otros 5 . En los estudios en animales de experimentación, se constató que la administración de DMF se asoció a la hiperplasia de los túbulos renales, a la vacuolización tubular grave y a una leve degeneración tubular. Si bien la dosis tóxica oral de DMF es de $2.250 \mathrm{mg} / \mathrm{kg}$ en los ratones, los estudios con dosis de $30 \mathrm{mg} / \mathrm{kg} / \mathrm{día}$ (entre 3 y 9,5 veces la dosis diaria) no mostraron evidencia de efectos adversos ${ }^{21}$. Por otro lado, Balak et ál. describieron 11 casos de disfunción tubular renal proximal, también conocido como síndrome de Fanconi, en mujeres en tratamiento con DMF durante más de 5 años ${ }^{5,22}$.

Los datos retrospectivos de efectos adversos en Alemania no describen un aumento del riesgo de enfermedades neoplásicas o infecciosas. Sin embargo, se recomienda evitar la prescripción conjunta de DMF con inmuno- 


\begin{tabular}{|c|c|}
\hline Generales & Frecuentes: fatiga, astenia y sofocos \\
\hline $\begin{array}{l}\text { Gastrointesti- } \\
\text { nales }\end{array}$ & $\begin{array}{l}\text { Muy frecuentes: diarrea, náuseas, distensión y } \\
\text { dolor abdominal } \\
\text { Frecuentes: vómitos, dispepsia, estreñimiento, } \\
\text { flatulencia, reducción del apetito, elevación de las } \\
\text { enzimas hepáticas }\end{array}$ \\
\hline Hematológicos & $\begin{array}{l}\text { Muy frecuentes: leucopenia, linfopenia } \\
\text { Frecuentes: eosinofilia, leucocitosis } \\
\text { Muy infrecuente: leucemia linfática aguda, panci- } \\
\text { topenia irreversible }\end{array}$ \\
\hline Neurológicos & $\begin{array}{l}\text { Frecuentes: cefaleas, parestesias } \\
\text { Ocasionales: mareos } \\
\text { Desconocidos: leucoencefalopatía multifocal } \\
\text { progresiva }\end{array}$ \\
\hline Dermatológicos & $\begin{array}{l}\text { Frecuentes: rubefacción, quemazón y prurito } \\
\text { (vía oral), dermatitis de contacto y urticaria por } \\
\text { contacto no inmunitaria (vía cutánea) } \\
\text { Infrecuentes: exantema macular }\end{array}$ \\
\hline Nefrológicos & $\begin{array}{l}\text { Ocasionales: proteinuria, elevación de la creatinina } \\
\text { sérica } \\
\text { Desconocidos: insuficiencia renal aguda, síndrome } \\
\text { de Fanconi }\end{array}$ \\
\hline \multicolumn{2}{|c|}{$\begin{array}{l}\text { TABLA 4: Efectos adversos del dimetilfumarato. } \\
\text { Muy frecuente: }>10 \% \text { de los pacientes; frecuente: } 1-10 \% \text {; casional: } 0,1-1 \% \text {; infrecuente: } 0,01 \text { - } \\
0,1 \% \text {; muy infrecuente: }<0,01 \% \text {; desconocidos: no se conoce su frecuencia. }\end{array}$} \\
\hline
\end{tabular}

supresores y, en caso de las vacunas elaboradas con virus vivos atenuados, suspender DMF y reiniciarlo después de 2 semanas $^{5,23}$.

La leucoencefalopatía multifocal progresiva (LMP) es un efecto adverso temido, aunque muy infrecuente, asociado al tratamiento con DMF. La LMP es una enfermedad desmielinizante asociada al poliomavirus JohnCunningham (JC) y caracterizada por la destrucción de los oligodendrocitos. En los pacientes en tratamiento con DMF, se destacaron como principales factores predisponentes de LMP la leucocitopenia moderada o grave, el uso de inmunosupresores antes del inicio de DMF y las enfermedades que comprometen la inmunidad como el $\mathrm{HIV} /$ sida, las enfermedades oncohematológicas y el lupus eritematoso, entre otras ${ }^{5,24}$. Gieselbach et ál. describieron el inicio de los síntomas desmielinizantes luego de una media de 31 meses de utilización de DMF, asociados a una linfocitopenia T CD4 absoluta $<200$ células $/ \mu 1^{25}$.

\section{CONTRAINDICACIONES DEL DIMETILFUMARATO, EMBARAZO Y LACTANCIA}

Dados los efectos adversos descriptos, las enfermedades gastrointestinales graves son una contraindicación debido a la baja tolerabilidad de DMF. Asimismo, las en- fermedades hepáticas y renales graves limitan su uso. El DMF está aprobado solo para pacientes mayores de 18 años y no debe haber alergias conocidas a alguno de sus compuestos ni utilizar otros derivados sistémicos o tópicos de EAF de forma simultánea ${ }^{5}$.

La administración de DMF durante el embarazo y la lactancia no está aprobada. A nivel experimental en ratones, DMF atraviesa la barrera placentaria, pero no se demostraron malformaciones congénitas, aunque en dosis de $40 \mathrm{mg} / \mathrm{kg} /$ día se describieron casos de lóbulos hepáticos supernumerarios y alineación ilíaca anormal. El registro de mujeres tratadas con DMF durante el primer trimestre de la gestación es escaso y no se constataron malformaciones congénitas 5 . Otros autores describieron un caso de transposición de los grandes vasos y otro, de estenosis pilórica en 54 recién nacidos vivos de madres con esclerosis múltiple que recibieron DMF en el primer trimestre ${ }^{26}$. No existen datos respecto de la exposición de DMF durante el segundo y el tercer trimestre o de su presencia en la leche materna, por lo que no se recomienda su administración durante estos períodos 5 .

\section{ESTUDIOS CLÍNICOS EN LA PSORIASIS}

En 2017 se publicó el estudio multicéntrico, aleatorizado y doble ciego BRIDGE, llevado a cabo en Austria, Alemania, Polonia y Países Bajos. Este estudio en fase III reclutó a 671 pacientes diagnosticados con psoriasis moderada a grave, que no recibieron tratamiento durante un ańo. Se distribuyó a los pacientes en tres ramas: DMF, EAF/DMF (Fumaderm ${ }^{\circledast}$ ) y placebo, en una proporción de 2:2:1 durante 16 semanas. La dosis máxima diaria de DMF fue de $720 \mathrm{mg}$, aunque ajustable según la tolerancia y la respuesta individual de los pacientes. Como objetivo, se midieron el PASI 75 y el PGA. Los resultados fueron estadísticamente significativos y los autores señalaron un PASI 75 alcanzado para DMF y EAF/DMF de 37,5\% y 40,3\%, respectivamente, mientras que para el del placebo fue solo de $15,3 \%$. Asimismo, el PGA alcanzado como nulo o mínimo fue de $33 \%$ para DMF, $37,4 \%$ para EAF/DMF y $13 \%$ para el placebo. La frecuencia de efectos adversos fue mayor en el grupo tratado con drogas activas que con el placebo $(83,9 \%$ y $84,1 \%$ contra $59,9 \%)$, si bien la mayoría de los que se describieron se encontraron dentro de la categoría de efectos adversos gastrointestinales leves. Al finalizar el tratamiento, se observó un rebrote de la psoriasis de 1,1\% para DMF, 2,2\% para EAF/DMF y $9,3 \%$ en los pacientes que recibieron el placebo. Los autores concluyeron que no existen diferencias significativas de eficacia y efectos adversos entre EAF/DMF y DMF y comprobaron que el principio activo DMF es esencial para el efecto terapéutico en la psoriasis ${ }^{27}$. 
cutáneo y es algo menor que el de la ciclosporina. Sin embargo, la relación costo-beneficio del DMF en la Argentina no lo hace atractivo como primera línea de tratamiento en la psoriasis, debido a que su costo es mayor que otras medicaciones orales con una eficacia similar. En el análisis comparativo entre metotrexato y EAF llevado a cabo por Inzinger et ál. se constató una eficacia similar entre ambas medicaciones (PASI 90: 5\% y PASI 75: 27\% contra metrotexato: PASI 100: 6\%, PASI 90: 7\% y PASI 75: 24\%) y una tasa semejante de suspensión por efectos adversos dentro del primer trimestre de tratamiento (EAF 41,7\% contra metotrexato 42,5\%). En la Tabla 5 se cotejan los tratamientos sistémicos en la psoriasis basados en su eficacia, nivel de evidencia y practicabilidad $^{28,29}$.

\section{UTILIZACIÓN DEL DIMETILFUMARATO EN PEDIATRÍA}

A nivel pediátrico, los EAF se han utilizado con indicación off-label en el tratamiento de la psoriasis moderada a grave. El estudio retrospectivo KIDS FUTURE, llevado a cabo en Alemania, recopiló información sobre la eficacia y seguridad a largo plazo de los EAF en 127 pacientes de entre 6 y 17 años de edad. El 75,6\% de esta población presentó psoriasis vulgar, el 59\% recibió una medicación tópica concomitante (corticosteroide, vitamina $\mathrm{D}$ o ácido salicílico) y la media del PASI al inicio del tratamiento fue de 17,3 . Si bien se utilizaron dosis crecientes de la medicación según se especificó anteriormente, la dosis media máxima de DMF no superó los $480 \mathrm{mg}$ (4 comprimidos diarios de Fumaderm ${ }^{\oplus}$ ). La eficacia terapéutica se observó entre el tercero y el sexto mes de tratamiento, con una reducción del BSA de 18,2 a 6,6 y del PGA de 2,7 a 1,3. El PASI 75 se alcanzó en el 18,9\% de los pacientes al tercer mes y en el 30,4\% a los 6 meses, mientras que en el PASI 90 fue de $7,6 \%$ y de $10,9 \%$ en los respectivos meses. Cerca del $30 \%$ de los pacientes presentaron efectos adversos, casi siempre leves, entre los que se destacaron los trastornos gastrointestinales y el flushing cutáneo como los más frecuentes. A nivel laboratorial, se encontró una leucocitosis transitoria durante los primeros 3 meses de tratamiento, con una posterior reducción por debajo de los valores basales, junto con una reducción del recuento de linfocitos y un aumento de GGT. Estos últimos dos parámetros se estabilizaron después de los 24 meses de tratamiento con Fumaderm ${ }^{\circledast}$. Las guías alemanas de manejo pediátrico de la psoriasis sugieren el uso de EAF en los pacientes con enfermedad moderada a grave con contraindicación al metotrexato o al adalimumab, o en quienes estos fármacos fueron ineficaces ${ }^{30}$.

\section{COMENTARIOS}

El DMF es una opción atractiva para el tratamiento en la psoriasis moderada a grave en los pacientes que realizaron terapéuticas tópicas y fototerapia sin tener mejoría o en los que no son candidatos para recibir otros tratamientos sistémicos. Como ventajas, la medicación requiere controles de laboratorio trimestrales, la dosis se ajusta según las necesidades del paciente, no se considera un medicamento inmunosupresor y la tasa de efectos adversos graves es muy baja. Si bien es una medicación costosa, poco difundida y no está autorizada en la Argentina para el tratamiento de la psoriasis, se destaca el interés por su conocimiento como alternativa terapéutica y se resaltan como particulares sus mecanismos de acción.

\begin{tabular}{|c|c|c|c|c|c|c|}
\hline Medicación & Eficacia ${ }^{(a)}$ & $\begin{array}{c}\text { Calidad de la eviden- } \\
\text { cia PASI } 75 \text { contra } \\
\text { placebo }\end{array}$ & $\begin{array}{l}\text { Seguridad y tole- } \\
\text { rancia durante la } \\
\text { inducción }\end{array}$ & $\begin{array}{l}\text { Seguridad y tole- } \\
\text { rancia durante el } \\
\text { mantenimiento }\end{array}$ & $\begin{array}{c}\text { Practicabili- } \\
\text { dad (paciente) }\end{array}$ & $\begin{array}{l}\text { Practicabili- } \\
\text { dad (médico) }\end{array}$ \\
\hline Acitretina & $0 /+$ & $\begin{array}{c}\text { No hay estudios } \\
\text { comparativos }\end{array}$ & + & + & + & ++ \\
\hline Adalimumab & +++ & +++ & ++ & ++ & +++ & ++ \\
\hline Apremilast & + & +++ & ++ & ++ & +++ & +++ \\
\hline Ciclosporina & + & ++ & + & + & +++ & ++ \\
\hline Etanercept & ++ & ++++ & ++ & ++ & +++ & ++ \\
\hline EAF & + & ++ & + & ++ & ++ & ++ \\
\hline Infliximab & ++++ & ++ & + & ++ & +++ & $+/-$ \\
\hline Metotrexato & + & ++ & + & ++ & +++ & ++ \\
\hline Secukinumab & ++++ & ++++ & ++ & ++ & +++ & ++ \\
\hline Ustekinumab & +++ & +++ & ++ & ++ & ++++ & +++ \\
\hline
\end{tabular}




\section{ABREVIATURAS}

ERK: extracellular signal-regulated kinase, quinasa regulada por señal extracelular.

HCA2: hydroxycarboxylic acid receptor 2, receptor de ácido hidroxicarboxílico 2 .

HIF-1 $\alpha$ : hypoxia inducible factor- $1 \alpha$, factor inducible por hipoxia $1 \alpha$.

IL: interleucina.

JAK/STAT: Janus Kinase/Signal Transducers and Activators of Transcription, Janus Kinasa/transductores de señal y activadores de transcripción.

\section{BIBLIOGRAFÍA}

1. Martinez Lombardo MA, Cano Ortiz A. Plantas medicinales con alcaloides en la provincia de Jaén. Boletín del Instituto de Estudios Giennenses 2009;200:125-163.

2. Rotering F. Ésteres de ácido fumárico (Fumaderm) en psoriasis. Arznei-Telegramm 2013;44:35-36.

3. Balak DM. Fumaric acid esters in the management of psoriasis. Psoriasis (Auckl) 2015;5:9-23.

4. Silvestre JF, Mercader $P$, Giménez-Arnau AM. Dermatitis de contacto por dimetilfumarato. Actas Dermosifiliogr 2010;101:217222.

5. Skilarence ${ }^{\circledast}$. Anexo I. Ficha técnica o resumen de las características del producto. [en línea], Agencia Europea de Medicamentos. 23 de junio 2017. Disponible en: <https://ec.europa.eu/health/ documents/community-register/2017/20170623138037/ anx_138037_es.pdf $>$ [consulta: abril 2020].

6. Brück J, Dringen $\mathrm{R}$, Amasuno A, Pau-Charles I, et ál. A review of the mechanisms of action of dimethylfumarate in the treatment of psoriasis. Exp Dermatol 2018;27:611-624.

7. Ceglowska U, Wlodarczyk A, Slomka M. Clinical effectiveness of fumaric acid esters (Fumaderm) in psoriasis: A systematic review of literature. Value Health 2014;17:A605.

8. Disposición 9666 [en línea], Administración Nacional de Medicamentos, Alimentos y Tecnología Médica, 26 de agosto de 2016. Disponible en: <http://www.anmat.gov.ar/boletin_anmat/ agosto_2016/Dispo_9666-16.pdf> [consulta: mayo 2020].

9. Meissner M, Valesky EM, Kippenberger S, Kaufmann R, et ál. Dimethyl fumarate - only an anti-psoriatic medication? J Dtsch Dermatol Ges 2012;10:793-801.

10. Blair HA. Dimethyl Fumarate: A Review in Moderate to Severe Plaque Psoriasis. Drugs 2018;78:123-130.

11. Rostami-Yazdi M, Clement B, Schmidt TJ, Schinor D, et ál. Detection of metabolites of fumaric acid esters in human urine: implications for their mode of action. J Invest Dermatol 2009;129:231-234.

12. Ghoreschi K, Brück J, Kellerer C, Deng C, et ál. Fumarates improve psoriasis and multiple sclerosis by inducing type II dendritic cells. J Exp Med 2011;208:2291-2303.

13. Lehmann JC, Listopad JJ, Rentzsch CU, Igney $\mathrm{FH}$, et ál. Dimethylfumarate induces immunosuppression via glutathione depletion and subsequent induction of heme oxygenase 1. J Invest Dermatol 2007;127:835-845.

14. Gillard GO, Collette B, Anderson J, Chao J, et ál. DMF, but not other fumarates, inhibits NF-KB activity in vitro in an Nrf2-independent manner. J Neuroimmunol 2015;283:74-85.

15. Litjens $\mathrm{NH}$, Rademaker $\mathrm{M}$, Ravensbergen $\mathrm{B}$, Rea $\mathrm{D}$, et ál. Monomethylfumarate affects polarization of monocyte-derived dendritic cells resulting in down-regulated Th1 lymphocyte responses. Eur J Immunol 2004;34:565-575.

16. Vandermeeren $M$, Janssens $S$, Borgers $M$, Geysen J. Dimethylfumarate is an Inhibitor of cytokine-induced E-selectin, VCAM-1, and ICAM-1 expression in human endothelial cells.

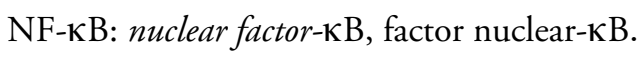

Nrf2: nuclear respiratory factor 2, factor respiratorio nuclear 2.

PASI: Psoriasis Area and Severity Index, índice de la severidad del área de psoriasis.

PGA: Physician Global Assessment, evaluación global del médico.

STAT3: Signal Transducers and Activators of Transcription 3, transductores de señal y activadores de transcripción 3.

VEGF: vascular endothelial growth factor, factor de crecimiento derivado del endotelio vascular.

Biochem Biophys Res Commun 1997;234:19-23.

17. Peng $\mathrm{H}$, Guerau-de-Arellano M, Mehta VB, Yang Y, et ál. Dimethyl fumarate inhibits dendritic cell maturation via nuclear factor $\mathrm{KB}$ (NF-KB) and extracellular signal-regulated kinase 1 and 2 (ERK1/2) and mitogen stress-activated kinase 1 (MSK1) signaling. $J$ Biol Chem 2012;287:28017-28026.

18. Zhao G, Liu Y, Fang J, Chen Y, et ál. Dimethyl fumarate inhibits the expression and function of hypoxia-inducible factor-1a (HIF-1a). Biochem Biophys Res Commun 2014;448:303-307.

19. Li Y, Tang J, Hu Y. Dimethyl fumarate protection against collagen II degradation. Biochem Biophys Res Commun 2014;454:257-261.

20. Schmitz L, Oster-Schmidt C. Ésteres de ácido fumárico. En: Schmitz L, Oster-Schmidt C. Terapéutica sistémica en la dermatología. Thieme, Stuttgart, 2016:195-198.

21. Safety Data Sheet - Dimethyl fumarate. [En línea], Thermo Fischer Scientific, Estados Unidos, enero de 2018. Disponible en: <https:// www.fishersci.com/> [consulta: mayo 2020].

22. Balak DM, Bouwes Bavinck JN, De Vries AP, Hartman J, et ál. Druginduced Fanconi syndrome associated with fumaric acid esters treatment for psoriasis: a case series. Clin Kidney J 2016;9:82-89.

23. Mrowietz U, Barker J, Boehncke WH, Iversen L, et ál. Clinical use of dimethyl fumarate in moderate-to-severe plaque-type psoriasis: a European expert consensus. J Eur Acad Dermatol Venereol 2018;32 Suppl 3:3-14.

24. Reich K, Thaci D, Mrowietz U, Kamps A, et ál. Efficacy and safety of fumaric acid esters in the long-term treatment of psoriasis-a retrospective study (FUTURE). J Dtsch Dermatol Ges 2009;7:603-611.

25. Gieselbach RJ, Muller-Hansma AH, Wijburg MT, De Bruin-Weller MS, et ál. Progressive multifocal leukoencephalopathy in patients treated with fumaric acid esters: a review of 19 cases. J Neurol 2017;264:1155-1164.

26. Everage NJ, Liu S, Yun J, Prada C, et ál. 053 Pregnancy outcomes with delayed-release dimethyl fumarate: interim results from an international registry. J Neurol Neurosurg Psychiatry 2018;89:A22.

27. Mrowietz U, Szepietowski JC, Loewe R, Van de Kerkhof $P$, et ál. Efficacy and safety of LAS41008 (dimethyl fumarate) in adults with moderate-to-severe chronic plaque psoriasis: a randomized, double-blind, Fumaderm ${ }^{\circledR}$ - and placebo-controlled trial (BRIDGE). Br J Dermatol 2017; 176:615-623.

28. Inzinger M, Weger W, Heschl B, Salmhofer W, et ál. Methotrexate vs. fumaric acid esters in moderate-to-severe chronic plaque psoriasis: data registry report on the efficacy under daily life conditions. J Eur Acad Dermatol Venereol 2013;27:861-866.

29. Nast A, Amelunxen L, Augustin M,WHBoehncke et ál. S3 Guideline for the treatment of psoriasis vulgaris, update - Short version part 1 - Systemic treatment. J Dtsch Dermatol Ges 2018;16:645-669.

30. Reich K, Hartl C, Gambichler T, Zschocke I. Retrospective data collection of psoriasis treatment with fumaric acid esters in children and adolescents in Germany (KIDS FUTURE study). J Dtsch Dermatol Ges 2016;14:50-58. 